INTERNATIONAL DESIGN CONFERENCE - DESIGN 2018

https://doi.org/10.21278/idc.2018.0136

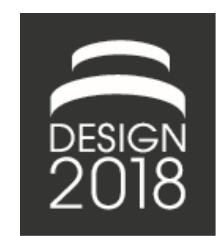

\title{
TOUCH, TOUCH, TOUCH, SENSORIAL COGNITIVE SKILLS SENSITIZED THROUGH TACTILITY AND TANGIBILITY
}

\author{
R. Wendrich
}

\begin{abstract}
This paper presents the development and testing of a tangible user interface (TUI) project that includes the exploration of haptics, design processes, hybrid design tools and unconventional user interfaces (NUI) that focus essentially on the tangible bits, embodiment, meta-cognitive and user interaction (IxD) on concrete devices designed to explore sensorial feedbacks (e.g. sense of touch, tactility etc.). We include preliminary user-testing with a heterogeneous group of 24 participants and show initial results and findings based on usability, interaction modality and user experience (UX).
\end{abstract}

Keywords: computational design methods, conceptual design, cyber physical systems, design creativity, uncertainty

\section{Introduction}

Domain shifts in design and /or engineering are the metamorphoses of fundamental change to evoke new or refreshingly different experiences. Metamorphosis can be orderly, disorderly or chaotic. For example; a foundational craft is cooking rather than weaving, potting or carpentry, but the logic of change applies to all crafts. Crafts is not design, however design could become a craft and one could become a crafty designer and/or engineer in such. Lévi-Strauss (1966) presents change as a culinary triangle; "...a triangular semantic field whose three points corresponds to the categories of the raw, the cooked, and the rotted." The raw is the realm of nature, as human beings find it: cooking creates the realm of culture, nature metamorphosed. It is always about culture, never about civilization. In cultural production food is both good to eat and good to think. Hence, from heating to sharing food, from eating to thinking instigating a domain shift. Metamorphosis provokes material consciousness in three ways: through internal evolution of type-form, in the judgment about mixture and synthesis, by the thinking involved in domain shift (Sennett, 2008). Flusser (1999) connects 'design' to all culture and states: '...that 'design' deceives nature by means of technology, to replace what is natural with what is artificial and build a machine out of which there comes a "god" who is ourselves.' Harrari (2016) follows in his footsteps and argues, '...that humans seek bliss and immortality but, are in fact trying to upgrade themselves into gods,...to acquire godlike control of their own biological substratum...to design and engineer our system in almost any manner we like...'

Hence, we could argue, that the shift, step-change, transformative variances lead to metamorphosis from analogue to digital design with the introduction of computational assistants and support systems should be regarded as a domain shift within the culture of design creating novel experiences in production and processes. 'We must learn how to handle these metamorphic systems effectively; but at the same time we must preserve and, if necessary, intensify our ability to look at the world directly and not through that half-opaque medium of synthetic system-concepts, which distorts every given fact into the all 
familiar likeness of some generic label, monolithic, or explanatory abstraction (Huxley, 1954)'. Whitehead (1985) explains the higher phases of experience, a simple physical feeling is the most primitive type of an act of perception, devoid of consciousness. In effect these feelings constitute the machinery by reason of which the creativity transcends the world already actual, and yet remains conditioned by that actual world in its new impersonation. Conscious perception is the feeling of what is relevant to immediate fact in contrast with its potential irrelevance. An affirmative intuitive judgement is very analogous to a conscious perception. A simple physical feeling (i.e. pure physical- and hybrid physical feelings), is an act of causation and enjoys a characteristic which has been variously described as 're-enaction,' 'reproduction,' and 'conformation.' The separation of body and mind is therefore disastrous (Whitehead, 1985; Johnson, 2013). The actual entity which is the initial datum is the 'cause,' the simple physical feeling is the 'effect,' and the subject entertaining the simple physical feeling is the actual entity 'conditioned' by the effect. This 'conditioned' actual entity will also be called the 'effect' (Whitehead, 1985). Impulsions are the beginnings of complete experiences because they proceed from need: from a hunger and demand that belongs to the organism as a whole and that can be supplied only by instituting definite relations (active relations, interactions) that wits the environment (Dewey, 2005). A pure conceptual feeling in its first mode of origination never involves consciousness, in this a pure mental feeling, conceptual or propositional, is analogous to a pure physical feeling (e.g. impressions of sensation). Vagueness has its origin in transmuted feelings. Consciousness arises when a synthetic feeling integrates physical and conceptual feelings (Whitehead, 1985). Music is the highest of the arts, because it gives us not merely the external objectifications of will but also sets before us for contemplation the very processes of will (i.e. a mode of revelation of the inner nature of things).

\section{Rawshaping technology (RST) approach}

RST research (Wendrich, 2009-2016) is on early-phase design processes, ideation, and abstract conceptualization in conjunction with the design, build and development of hybrid design tools (HDT) that assist and support externalization, representation and visualization of "ideas" (Brockman, 2015) informed by tacit and/or embodied skills and knowledge (Polanyi, 1966; Collins, 2010; Grant, 2011). This allows users to interact and reflect unconstraint and randomly on raw ideas, fuzzy notions, lively dreams or unfocused thoughts. HDT's afford designers to be, to instil and to become creative, curious and explorative to address uncommon ideas they might recognize as having potential or wit. To paraphrase Kierkegaard (1813-1855); 'Fixed ideas are like a cramp in the foot-the best remedy is to thread on it.' Current times (as ever before) call for ideas, however ideas change, and the times we live in change. Perhaps the biggest change today is the rate of change. What scientific idea is ready to be moved aside so that science can advance (Brockman, 2015)? Everything, every...thing?

\subsection{Usability, tacit knowledge, embodied interaction and reflection}

According to Polanyi (1966) in order to gain knowledge we process our experiences and sensorial input to form comprehensive entities. By combining perceptions with non-perceived information more abstract and complex comprehensive entities are formed. This is how we can recognize a face without being aware of all of its elements. Knowledge gained from learning experiences is harder to express the more complex it becomes. According to Grant (2011) "...tacitness is something personal, an ability or skill to do something or to resolve a problem that is based, in part, on one's own experiences in learning". Polanyi (1966) differentiates between proximal and distal tacit knowing, just like we perceive the world through our body, we perceive an artefact (digital or physical) we are working on through the tool we are working with. When one becomes proficient at using a tool, like e.g. a bicycle the focus shifts from how one is sitting on and balancing the bike to staying on path while moving. The same thing happens in a creative process when one is in a state of flow. From the work of Csikszentmihalyi (1990) we understand that flow is a state in-between unselfconscious immersion during performance of a task and reflection-in-action (Schön, 1983) leading to fast choice and decision-making. Other forms of reflection informed by tacit knowing in a creative process are reflection-in-conversation, reflection-inpractice (specialization through repetition of actions) and reflection-on-action (after the action was performed). 


\subsection{Hybrid design tools and usability}

Usability in an artefact or system is defined by its affordances and the sequence of actions needed to achieve an affordance. Affordances in interaction design (IxD) are what an artefact or system allows its user to do, understand, see, hear, feel or taste. How difficult performing an action with a tool/artefact is perceived to be depends heavily on how familiar the user is with performing the action to achieve a certain thing. No interface is truly intuitive in the sense that the user does not need any knowledge to use an artefact (Spool, 2005). By interacting with the world around them people learn how to interact with it. When an interaction is built on this tacit knowledge it is perceived to be intuitive. Usability and visualisation with for example CAD systems is often perceived to produce unsatisfying results and create deficiency (Bilda and Gero, 2005; Robertson and Radcliffe, 2009; Kosmadoudi et al., 2013). They often require the user to perform a complex sequence of steps that have little to do with the actions performed in a physical design process and are thus not familiar. This results in a high threshold in learning curve. Similar complaints apply to virtual digital music production tools like for example Ableton Live and Logic Pro. While they often offer great affordances that are not possible to achieve with physical instruments, there is a persistence in high threshold learning curve that as a consequence do not foster fast, intuitive exploration of raw ideas. Wendrich (2012, 2014 and 2016) proposes improving on the deficiencies in virtual design tools by combining the beneficial factors of physical and virtual representation. "We need to develop tools that allow the same subtle physical freedom and gestural motions traditional tools and instruments embody. Tools that support intuitive expressiveness, creative flow, rapid prototyping, allow speedy interaction and give sensory feedback."

\subsection{Natural user interfaces (NUI)}

Jetter et al. (2014) stated that computers still demand special skills from users and distract them with secondary tasks, like configuration of settings. Instead they should offer immediate access to affordances "so that we are freed to use them without thinking and "mental gymnastics" and to focus beyond computers on new goals." They draw on the concept of conceptual integration to explain why computers can be very difficult to use. Computers are difficult to use because they require too many steps of conceptual integration and do not make enough use of simple concepts that most people have learned through interaction with the physical world and their social context (Jetter et al., 2014). Conceptual integration is the idea that humans learn complex concepts like 'shame' or 'happiness' through integration of other less complex concepts. This means that basic concepts that can be learned through perception like 'up' and 'down' are connected to complex concepts through many steps of integration (Lakoff and Johnson, 1980). This relates to theory on tacit knowledge, because one is mostly unconsciously using these concepts to carry out tasks. This also means that the mind has to be viewed as inherently embodied, because even the most abstract tasks are built on bodily experiences (Johnson, 1987). Furthermore, if WIMP interfaces are difficult to use, because they are too abstract, complex and 'unnatural', how can we design VE's that are perceived to be natural? When VE interaction is closer to interaction in the 'real' physical world, it becomes easier for users to understand and use digital technologies. Valli (2006) also sees the necessity of hybrid interaction tools and virtual representation of artefacts that can be manipulated similarly to physical artefacts. Both Wendrich et al. (2009), Valli (2006) propose the development of VEs that support natural interaction; "Natural interaction is defined in terms of experience: people naturally communicate through gestures, expressions, movements, and discover the world by looking around and manipulating physical stuff." To achieve spontaneous interaction through simplicity and physical interaction the representation of content, organization of information and the interactive device more and more simple and invisible (Weiser, 1993; Valli, 2006; Wendrich, 2010). As interactive technologies become more and more embedded and behave more like physical entities, users become less aware of the mediation taking place and the interaction becomes more fluid and natural. TP is offering a wide range of affordances through immediate, spontaneous hybrid interactions but strictly speaking is not a natural interface. Although it features physical interaction, the virtual output data is mapped to sounds that are either digitally created or sampled sounds that were created by different physical interactions and manipulations (Schaefer, 2016). 


\subsection{Tangible bits and intuitive tangible user interfaces (TUI)}

The Tangible Pods interface (TP-IF) (Figure 1) was developed for audio and haptic feedback, while RST focused on the development of HDT's for visual and haptic visualization, ideation and conceptualization in earlier and ongoing work. Theoretical background and framework references were implemented in the concept and final design of the TP-IF. The TP-prototype supports intuitive experimentation and expression in sound through tangible physical interaction. The interface could also be applied for combinatorial visual and audio feedback, however the focus is placed on synthesizing sound.
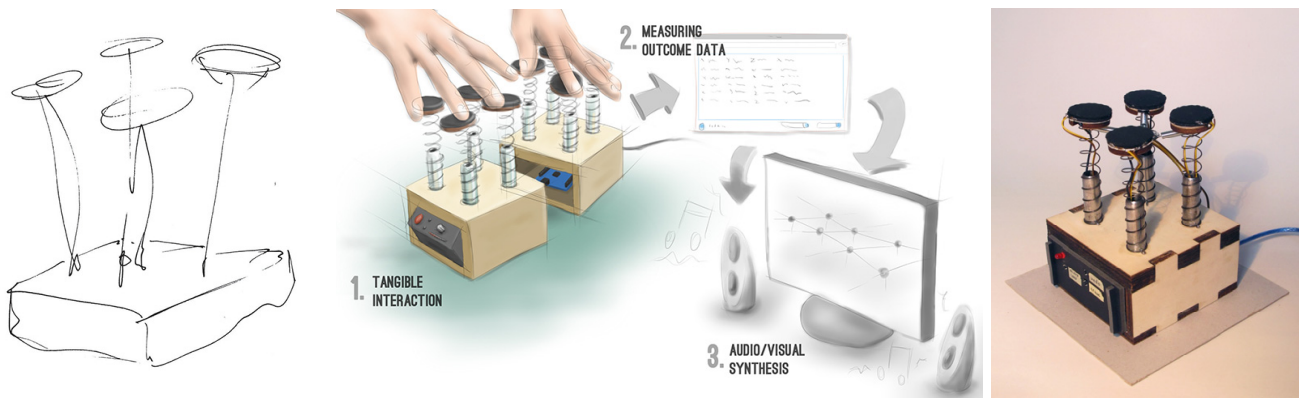

Figure 1. Tangible Pod interface and prototype

The interface should allow the user to think in sound, explore sound patterns, and experience auditorytactile synaesthesia that can induce sensations in parts of the body, evoke spatial awareness, and nudge users to explore uncommon sounds by introducing randomness into the process. In addition, it proposes improvement on some of the usability deficits of existing music production tools (i.e. Ableton Live and Logic Pro). Guidelines derived from Wendrich $(2010,2016)$ were applied and used for design and development of the TP-IF:

- Mediate between physical and virtual representations

- Support untethered bi-manual tangible interactions

- Facilitate tinkering and exploration of sound

- Facilitate a process of iteration, synthesis, and morphing

- Allow for immediate natural interaction

- Allow for intuitive expressiveness of the user

- Be intuitively usable and give the user a sense of being in control

- Facilitate enter into flow state

- Support thinking processes and decision making in sound production

- Support collaboration, conversation and back and forth signalling between multiple users using multiple tools

- Support ambiguity and randomness

- Support play, fun, and enjoyment

Ishii et al. (1997) coined the term tangible bits, digital data that can be accessed through interaction with tangible objects. They established three ways of bridging the gap between virtual environments (VE) and physical environments (PE):

- Interactive surfaces in an architectural environment that allow immediate access to a VE

- Hybrid artefacts that allow access to and manipulation of digital data belonging to a physical artefact

- Ambient media such as sound, light, and temperature that influence and are influenced by users

\subsection{Jamming user interfaces (JUI)}

JUIs are malleable and shape changing and perceived to be more organic than traditional interaction modalities, like mouse and keyboard or touchscreens. According to Follmer et al. (2012) there are two types of high-resolution shape sensing methods: Index-matched particles and fluids and capacitive and 
electric field sensing. They propose the development of interfaces that are not only deformable and stay in form but can also be computationally actuated to change their material properties like stiffness. They see that so far there have been more advances in shape sensing and mechanical actuation than in particle jamming, which is the computational actuation of material properties. The Tunable Clay JUI is characteristic, it uses a hydraulic system to change the material properties and structured light depth sensing to measure deformation of the interface. Other interfaces that allow for haptic feedback provide information for the user through shape-changing capabilities. Haptic feedback can be categorized into active and passive forms of feedback. Passive feedback is the feedback a material provides because of its properties, for example; foams, springs and rubbers return to their original state, if deformed to a certain degree. Active feedback, or actuation, is when an active mechanism changes the form of an interface. In mechanical actuation this is often done using electrical motors, as shown in Ros (2015) 'Who is Stewart', a tactile interface based on the Stewart platform that can move in six degrees of freedom (DoF), using six servomotors. It can also be used for haptic input, allowing for tactile communication between the user and the machine (Terken et al., 2017). Mechanical actuation and particle jamming provide new ways of providing feedback to users, mostly neglected in traditional UI's. Even though in many buttons springs are used to provide passive haptic feedback, the full potential of passive feedback modalities have not been explored in traditional UI's. Furthermore, passive feedback is easier to implement in interfaces, since no motors or pumps are needed. For the development of TP's a choice was made to explore passive feedback modalities.

\section{Case-study: Tangible tactile tinkering, testing and experimentation}

Over the years we have conducted a multiplicity in experimentation of human-machine interaction (HMI), testing a plethora of user interaction (UIA) modalities, user experiences (UX) and fundamental examinations and research in usability studies. Most of our work, in terms of approach, framework building, findings and results, is documented or in process of documentation. The following presents work carried out in the year 2014 to the beginning of 2015, it entails the design and build of a device called Tangible Pods (TP) described as a raw tangible user interface (TUI) for auditory and tangible tactile feedback. We have no statistical rituals (Gigerenzer, 2015), meaning the null ritual procedure (or p-values) that applied from the onset, during or after this case study. A ritual is a collective or solemn ceremony consisting of actions performed in a prescribed order (ibid. p. 524). However, not performing the ritual can provoke great anxiety, even when it makes absolutely no sense. So, in a sense we study rituals, not perform rituals ourselves. The objective of this test and experimentation is to explore, experiment and test diverse ways of creating tangible user-interfaces (TUI) that support visual, tactile and auditory interaction and afford feedback (i.e. cues, nudges) and assistive responses. In addition we study the possible and/or apparent impact of interaction modalities on embodied cognitive processes. The aim is to design a hybrid design tool (HDT) for physical-intuitive interaction within a virtual environment (VE) and to study embodied (physical) interaction in support of and assistance in metacognitive skilfulness and learning.

\subsection{Framework and foundation}

Computers mostly work with visual cues and windows, icons, menus and pointers (WIMP) interfaces and keyboard and mouse devices to interact with them (Beaudouin-Lafon, 2004). Tactile cues are sometimes used as well, but in less prominent and/or obvious ways. Recently, TUIs have been developed that integrate physical objects in virtual interaction to bridge the gap between the digital and physical realm (Shaer and Hornecker, 2010). While most graphical user interfaces (GUIs) are based on the desktop metaphor, the physical world offers a large amount of stuff in a plethora of contexts that one can physically interact with in many ways. However, the division between these two worlds is starting to crumble (Hornecker, 2011), because of TUIs among other things. TUIs allow users to create and interact with digital data in a physical way. They often support multisensory perception and make use of physical objects, instruments, architecture or spaces (Ullmer, 1997). The purpose of the TP-TUI is to foster and enrich abstract thinking processes through tangible-auditory interaction, externalization, and manipulation. 


\subsection{Tangible Pods (TP) concept as tangible user interface (TP-TUI)}

The TP consists of multiple "pods" that form a surface and can be physically manipulated/sculpted to create input data. It is a flexible interface that can be used in a range of applications including music, 2$\mathrm{D}$ and 3-D modeling (Figure 2).
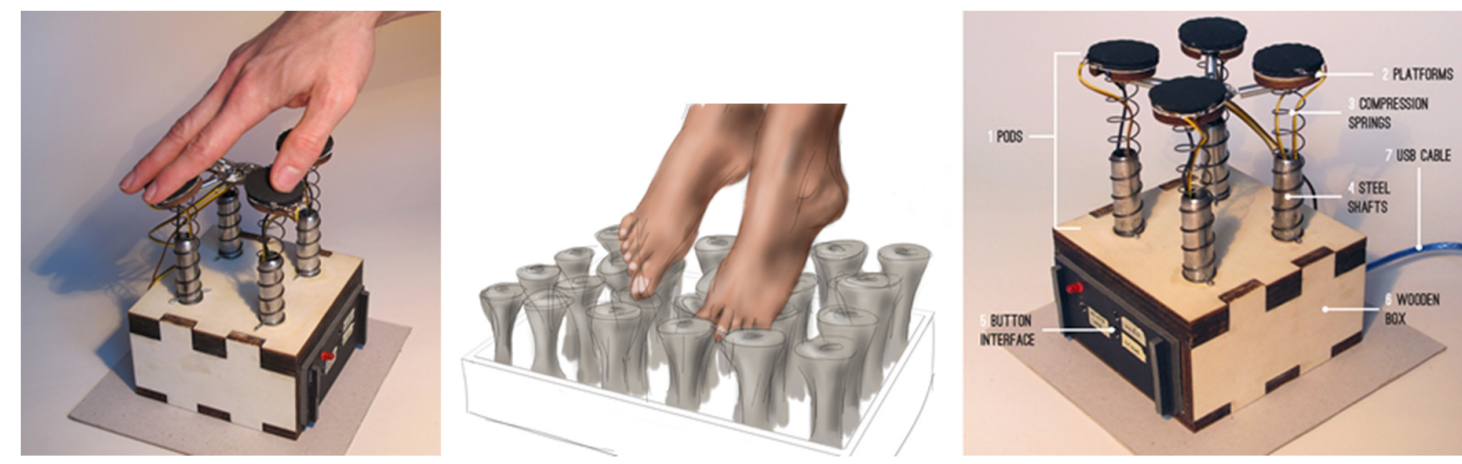

Figure 2. Tangible Pods concept and full functional prototype

The interface is made for instant creation and self-expression through sound and visuals using computer technology. It facilitates this by giving digital data a haptic manifestation. Similar to a computer mouse, a physical action measured by a sensor is interpreted by the computer as input that feeds back to the user; only in this case the interaction is three-dimensional and supports three axes of rotation (six degrees of freedom). The TP is a hybrid-tangible interface (HTI), that makes interaction more intuitive than traditional windows, icons, mouse, pointers (WIMP) (Van Dam, 1997; Jacob et al., 2008) interfaces since the user can rely more on his/her embodied and tacit knowledge of interacting with physical objects. Because of this affect, the TP can facilitate more fluid and easy participation of e.g. non-experts in the creation of electronic music, soundscape-sculpting or data-manipulation (e.g. tangible bits).

\subsubsection{Prototyping and conceptualization}

The final working prototype (Figure 2) consists of compression springs that are made from steel wire. These springs serve as direct tactile feedback when manipulated. Tactile-touch platforms in wood fixed atop the springs are covered with soft neoprene fabric and velostat material that again are sandwiched and assembled on top of the pressure sensors to create a tactile richness in experience. Pressure sensors, piezo's, and an accelerometer are used to measure the physical input and an Arduino microcontroller is used to process the resulting input data which can then be sent to a variety of digital music applications and/or programs (i.e. any that works with MIDI data). The prototype has four pods for practical reasons, however the number can be arranged and changed to fit the application's needs. Furthermore, the pods could also be embedded in an application that supports physical footwork creating interaction with one or both feet (Figure 2 center). Steel springs are better suited for being physically manipulated by the user, creating input-data (tilting, compression measured by sensors) and giving haptic feedback by going back to their original state after manipulation. Furthermore, springs are available in a wide range of elasticity and sizes, which makes them suitable for applications of various scale and types of interaction. A variety of novel interaction concepts emerge and eventually be scaled up to a size, where e.g. one pod has to be manipulated with the whole hand or scaled down to a size where it could be integrated with furniture for partial or full-body interaction. The prototype springs are most suited because of their relatively easy compression, even when pressed and/or twisted four at the same time. The springs are proportionally thin in wire and have a length of $100 \mathrm{~mm}$, a diameter of $17,5 \mathrm{~mm}$ and a wire-thickness of $1 \mathrm{~mm}$.

\subsubsection{Sensors, pods and buttons for interface interaction}

The sensors are key factor in the UX as they translate analog input into digital data. Many different kinds of sensors have been used for tangible interfaces (refs). Four piezo's (Murata 7BB-35-3), four 
pressure sensors and one accelerometer (Adafruit MMA8451triple-axis) have been used in the prototype. The piezo's pick up vibrations in the object they are attached to and used for detecting peaks in the vibration of the pods, to detect when the pod is being touched, manipulated and/or moved. Piezo's are cheap and relatively easy to implement, but not very precise. They can also pick up the frequencies of the vibrations and be used to determine where (location) and how (manipulation) an object is being touched, for more and precise data acquisition and analysis. At present this is not part of this particular case study. In addition to the piezos, pressure sensors are being used in the prototype instead of the flex sensors in previous test set-ups. Pressure and flex sensors work in a very similar way. When pressed/flexed the output voltage of the sensor is higher, because the resistance is lowered. When a pressure sensor (pod) is being pushed down, the higher the output value of the sensor is. The pressuresensors are hand-made instead of pre-fabricated, to make the prototype cost less, facilitate easier size and material (neoprene) propagation and made fit to purpose i.e. low cost-high value.

Lastly, an accelerometer was added to measure how the user is tilting/playing/manipulating the pods. For this prototype development, which is an approximation of a further developed artefact, using just one accelerometer is cheaper and sufficient to proof the principle. The accelerometer was placed on a small steel disc that is connected to all four pods with extension springs, so that it moves with the pods but does not limit their movement too much. Various arrangements and numbers of pods were considered and tested using just the compression springs. To make up an imagined surface that the user can place his palm on at least three pods are needed. The cost was calculated for all the parts for the different numbers of pods. Choosing four pods for the prototype seemed a good compromise. The pods were fixed and assembled onto a wooden box that contains the Arduino board (UNO r3), the 16-channel multiplexer (MUX) and an electronic circuit (Figure 2).

\section{Usability testing with integration of TP}

The TP interface should facilitate exploration of possibilties in digital sound synthesis through physical interaction. During synthesis the user should be able to arrange sounds into a composition (by e.g. looping them) and manipulate sound properties (e.g. timbre, amplitude, length, pitch). After that, the user should also be able to reflect on and change the composition and quality of the sound either through physical interaction or a simple button or screen-based interface. Difference in behavior of physical and digital artefacts might be counter-intuitive but will always exist with current technology. The resulting hybrid interface can still facilitate discovery, when the physical interaction is familiar but results in something unexpected. The interface facilitates interaction with computers that is more fluid and natural, but also less precise. The output data has to be interpreted by a computer and translated into sound in a meaningful way. The interface can produce a diverse range of sounds from varying contexts and with varying intensity, pitch, duration and timbre. The data acquired by the sensors could be used for visual (2-D/3-D) interaction as well and be implemented in design processing. The interface gives the user access to possibilities of the digital realm, which are otherwise only accessible by learning software that is developed for professionals and therefore often has a high threshold in learning curve. A wide range of people should be able to use the interface. Usability study including heterogeneous users was tested in conjunction with the TP-setup (Figure 2 and Figure 3). The test is based on the System Usability Scale (SUS) developed by Brooke (1996). He defines usability as an artefact's appropriateness to a purpose in terms of effectiveness, efficiency and satisfaction. SUS is used to indicate whether users can quickly learn how to use the prototype and whether they enjoy using the prototype.

\subsection{TP interaction with heterogeneous users $(n=13)$}

A preliminary usability study with heterogeneous users in conjunction with the novel TP computer interface was executed and based on the assumption that an average user of UIs do not exist. and Studying a plethora of different people could possibly enrich the design and development process and assists in developing interfaces that adapt to the user's needs instead of the other way around (https://vimeo.com/174633269). 

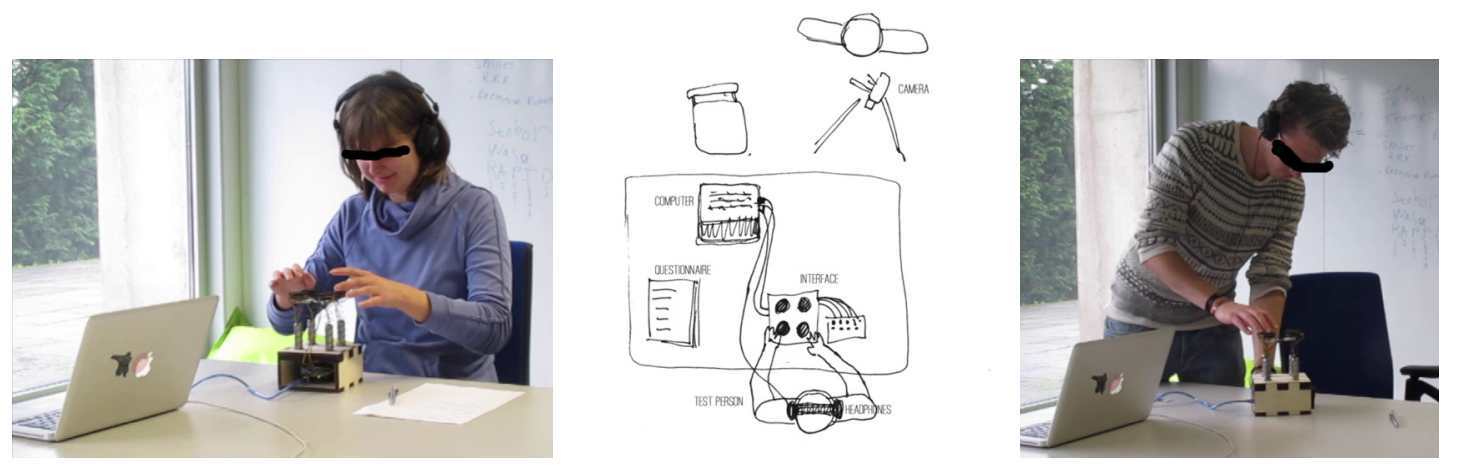

Figure 3. TP usability experimentation and typical test setup

For the design of a TUI (TP-IF), a choice was made to focus on embodied cognition and how the body's physicality influences psychological processes and iterative outcomes of interaction processes.

\subsubsection{Embodied cognition and HCI}

Antle et al. (2009) noticed a shift from viewing cognition as a disembodied processing of signals to a process that requires physical and mental activity. This shift to embodied cognition is one that should be taken into consideration in human computer interaction (HCI) notably following three ideas in embodied cognition:

- Meaning is created through spatial restructuring of elements in a (virtual) environment

- Physical actions can enhance and simplify cognitive tasks, e.g. physical manipulation can help learning the manipulation of mental models

- Bodily experiences can provide metaphors to help understanding abstract concepts, e.g. physical movement as a metaphor for progression in development ("look, how far I've come!")

These ideas can be implemented in the design of user interfaces (UI's). Computer interfaces should feature tangible structures, which offer affordances through physical manipulation and/or allow for manipulation of virtual representations. Similar to Wendrich (2012) proposal for hybrid tools that merge the beneficial factors of physical and virtual tools. Two examples for interfaces that implement object manipulation are the rawshaping formfinding hybrid design tool (RSFF-HDT) through manipulation of the object itself and the tangible programming space (Fernaeus and Tholander, 2006), which allows users to solve programming problems by changing the spatial location of objects (https://vimeo.com/43850666). Cognitive tasks can be simplified when physical interaction with a tangible is used as input. Antle et al. (2009) developed a hybrid physical VE that maps full-body input actions to musical sounds. In the development they found that tracing abstract cognitive processes from related bodily movements and using this for the mapping process improved the experience and performance of users. This suggests that more abstract thinking processes can partly be traced back from physical movements.

\subsubsection{User and usability testing}

Thirteen people $(\mathrm{n}=13)$ took part in the usability test. They are all between 20 and 34 years old and the average age of participants is ca. 24. Two of the thirteen participants are female and eleven are male. Five participants said they had no experience with making music, three said they had some and five said they had a lot of experience. No professional musicians participated. People of any age and gender were allowed to participate. The intended male/female division within the group of participants is 50/50.

\subsubsection{Procedure}

First, the participant is asked to state or fill out their age and gender. Then asked to put on headphones, play with the prototype by touching the interface and make sounds for approximately two minutes. After this one is asked to answer or fill out questions regarding using the interface like "did you find it difficult to use this interface? Then more information on how to use the interface and specifically the loop 
function is given and asked again to play with it for approximately 2 minutes. Then the participant is asked to fill in the similar questions a second time and in addition two open questions are posed. During the two 2-minute tests of the interface, video recording of the participant interaction is captured. The difference between the first and second test is that only in the second test the looping function has been introduced to the participants in order to determine whether this has an influence on the usability.

\subsubsection{Evaluation with SUS}

SUS (Likert scale) is used to determine the degree of agreement or disagreement with a statement 30 . The researcher then looks for general agreements or disagreements with certain statements in a group of participants. Furthermore, a usability score is calculated from each participant's answers to the questions based on the SUS. Answers to the open questions are used to determine possible improvements on the design through a simple, ten-item scale giving a global view of subjective assessments of usability (Brooke, 1996).

\subsubsection{SUS 1}

After using the prototype for the first time the participants were asked to fill out a SUS form. In this form they are given ten statements about the prototype to which they can respond on a scale of one to five from strong disagreement to strong agreement. The responses that express a positive judgment about the prototype are highlight green while responses that express a negative judgment are highlight red. Before filling out the second SUS form users were given a short instruction on how to use the looping-function of the prototype and were given some time to try it out. The statements in the second form did not elicit uniform agreement. This time, however, eight of the statements received more positive judgments about the prototype of which one received no negative judgment at all.

\subsubsection{Preliminary analysis on SUS and questionnaires}

To analyze both the written and the spoken reactions they were categorized into themes depending on what the reaction referred to. After that, reactions were further sorted into sub-themes and an overview was made of the amount of positive and negative reactions and observations regarding these sub-themes (Figure 4).

\begin{tabular}{|c|c|c|c|c|c|}
\hline Theme & Sub-theme & $\begin{array}{l}\text { Positive } \\
\text { experience }\end{array}$ & $\begin{array}{l}\text { Negative } \\
\text { experience }\end{array}$ & Observation & Total \\
\hline \multirow{2}{*}{ Pods } & Sensitivity & & 3 & 1 & 4 \\
\hline & $\begin{array}{l}\text { Number of } \\
\text { pods }\end{array}$ & & 1 & & 1 \\
\hline \multirow{2}{*}{$\begin{array}{l}\text { Erase'- and } \\
\text { unIdu'-butlun }\end{array}$} & $\begin{array}{l}\text { Undo erases } \\
\text { everything }\end{array}$ & & 2 & & 2 \\
\hline & $\begin{array}{l}\text { Undo stops } \\
\text { loop }\end{array}$ & & 1 & & 1 \\
\hline \multirow{3}{*}{$\begin{array}{l}\text { Audio feed- } \\
\text { back }\end{array}$} & Delay & & 2 & & 2 \\
\hline & Consistency & & 3 & & 3 \\
\hline & Precision & & 1 & & 1 \\
\hline \multirow{5}{*}{ Loop function } & $\begin{array}{l}\text { Display and } \\
\text { changing loop } \\
\text { length }\end{array}$ & & 3 & & 3 \\
\hline & Loop volume & & 1 & & 1 \\
\hline & $\begin{array}{l}\text { Keeping } \\
\text { rhythm }\end{array}$ & & 1 & & 1 \\
\hline & $\begin{array}{l}\text { Manipulating } \\
\text { sounds in hte } \\
\text { loop }\end{array}$ & & 1 & & 1 \\
\hline & $\begin{array}{l}\text { Missing play/ } \\
\text { pause button }\end{array}$ & & 1 & & 1 \\
\hline
\end{tabular}

\begin{tabular}{|c|c|c|c|c|c|}
\hline Theme & Sub-theme & $\begin{array}{c}\text { Positive } \\
\text { experience }\end{array}$ & $\begin{array}{l}\text { Negative } \\
\text { experience }\end{array}$ & Observation & Tota \\
\hline \multirow{2}{*}{ Sounds } & Variety & & 4 & & 4 \\
\hline & Differentiation & & 2 & & 2 \\
\hline $\begin{array}{l}\text { Visual appear- } \\
\text { ance }\end{array}$ & Colors & & 1 & & 1 \\
\hline \multicolumn{2}{|l|}{ Usability } & & 2 & & 2 \\
\hline \multirow{4}{*}{$\begin{array}{l}\text { General reac- } \\
\text { tion }\end{array}$} & $\begin{array}{l}\text { Mechanical } \\
\text { parts }\end{array}$ & 1 & 1 & & 2 \\
\hline & $\begin{array}{l}\text { Emotional } \\
\text { reaction }\end{array}$ & 4 & & & 4 \\
\hline & Purpose & & & 2 & 2 \\
\hline & Unusualness & & & 4 & 4 \\
\hline
\end{tabular}

Figure 4. Themes and sub-themes

\subsection{Analysis and evaluation}

The average SUS score from the second form was 69,8 a little higher than the first one. Scores ranged from 32,5 to 88 . The difference in average SUS score was approximately 9,7 while in the first form it was 
approximately 3,7. The second SUS scores (incl. loop functionality) are not consistently higher than the scores after first use (Table 1). The results from the answers open questions and other reactions did however result in a list of issues that can be improved to possibly enhance the usability of the TP-IF TUI:

- Sensibility of the pods

- Erasing sounds from the loop

- Delay between interaction and feedback

- Consistency in feedback
- Displaying loop length and allowing change

- Offer a larger variety of sounds

- Offer more distinct sounds/pod

Table 1. SUS 1 and 2 scores $(n=13)$

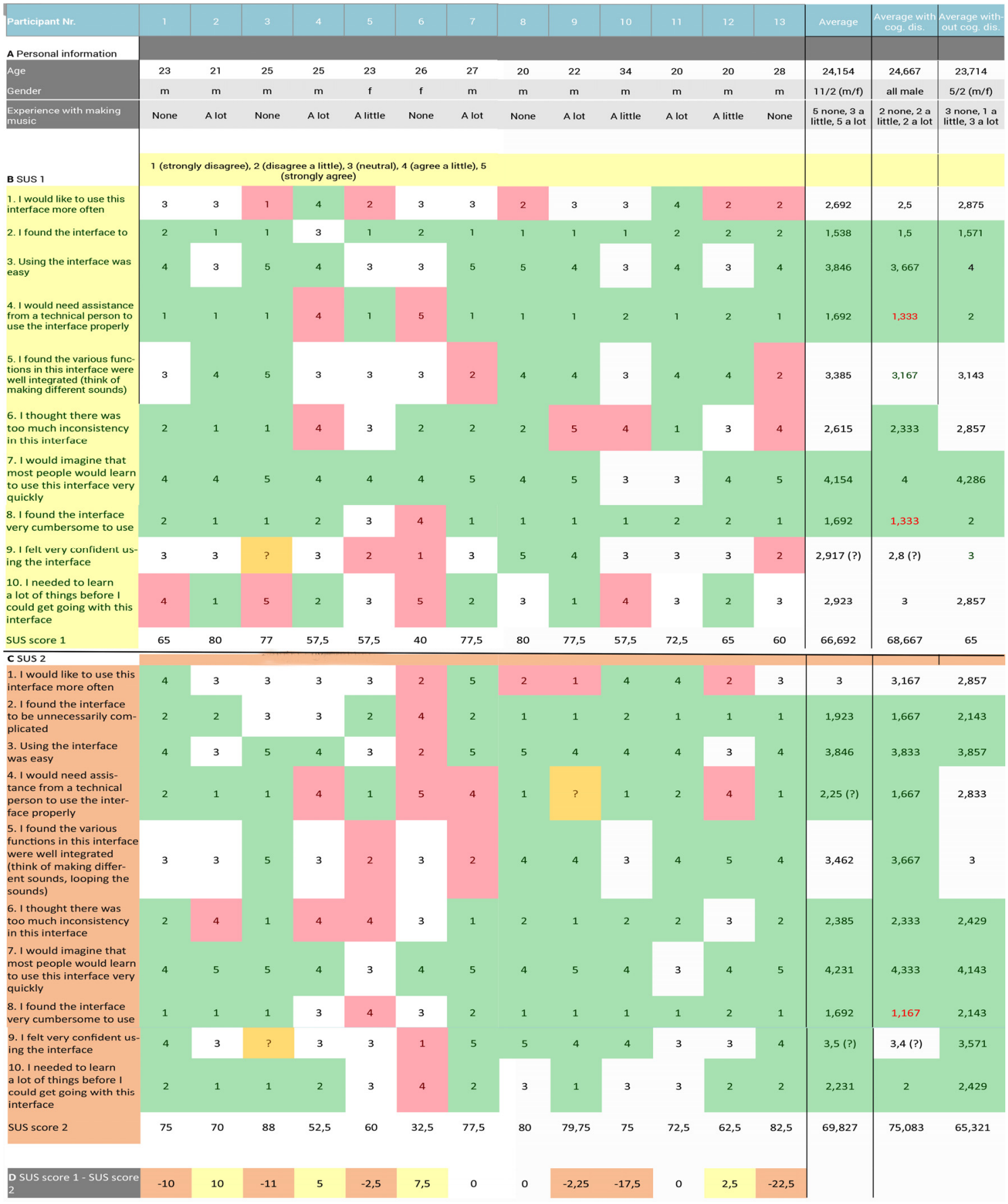




\section{Conclusion}

To conclude, humans compensates for his natural deficiencies by means of "umburdenings". Human's "eccentric position" compels him to make compensatory attempts at recentering: "Humans, wants to escape the unbearable eccentricity of his nature," and consequently "seeks compensation for his incompleteness, lack of equilibrium, nakedness," through culture: through technology, expressiveness, transcendence (Plessner in Marquard, 1991). TP's allow users to spontaneously and intuitively play with digital sounds, generate sound scapes, virtual 3-D models, support hybrid tangible interactions, affords haptic feedback and foster embodied thinking processes. The RST framework and process aim to design, develop and build interfaces that support humans in achieving their goals and/or tasks by making use of their capacities, capabilities, skill-set, embodied and explicit knowledge and integration through application of the virtual into the natural 'real' environment. More research needs to be done in order to make justifiable conclusions based on the data from the usability test. Further development of TP's could entail 3-D visual feedback; improving current functions of the prototype; better support in bi-manual interaction; and allowing multiple users to use the interface concurrently. The interface could also be adapted for other interactions like for example interaction using one's feet. After development of a new version of the interface, a study on the influence on behaviour and meta-cognitive skilfulness will be investigated. Most participants found the prototype to be relatively usable, no general agreement on any negative statement was formed. The interface with and without the loop function was perceived not of too much difference. Overall there is room for improvement, based on responses, number of complaints and suggestions that were made in response to the open questions.

\section{Acknowledgment}

The author likes to thank Peter Schaefer for his work and contribution to RST research.

\section{References}

Ableton, (n.d.), Max for Live. [online] Available at: https://www.ableton.com/en/live/max-for-live/ (accessed 08.12.2017)

Antle, A.N., Corness, G. and Droumeva, M. (2009), "What the body knows: Exploring the benefits of embodied metaphors in hybrid physical digital environments", Interacting with Computers, Vol. 21 No. 1, pp. 66-75.

Beaudouin-Lafon, M. (2004), "Designing interaction, not interfaces", Proceedings of the working conference on Advanced visual interfaces, ACM, pp. 15-22.

Bilda, Z. and Gero, J.S. (2005), "Do We Need CAD during Conceptual Design?”, Computer Aided Architectural Design Futures 2005, Springer Netherlands, pp. 155-164.

Brockman, J. (2015), This idea must die, Tantor Media.

Brooke, J. (1996), "SUS-A quick and dirty usability scale", Usability evaluation in industry, Vol. 189 No. 194, pp. 4-7.

Collins, H.M. (2010), Tacit and explicit knowledge, University of Chicago Press Ltd., Chicago, IL.

Csikszentmihalyi, M. (1990), Flow: The psychology of optimal performance, Cambridge University Press, NY.

Dewey, J. (2005), Art as experience, Penguin.

Fernaeus, Y. and Tholander, J. (2006), "Finding design qualities in a tangible programming space", Proceedings of CHI 2006, ACM Press, New York, pp. 447-456.

Flusser, V. (1999), The Shape of Things: A Philosophy of Design, Reaktion Books, London, U.K.

Follmer, S., Leithinger, D., Olwal, A., Cheng, N. and Ishii, H. (2012), "Jamming user interfaces: programmable particle stiffness and sensing for malleable and shape-changing devices", Proceedings of the 25th annual ACM symposium on User interface software and technology, ACM, pp. 519-528.

Gigerenzer, G. (2015), Risk savvy: How to make good decisions, Penguin.

Grant, K. (2011), “Tacit Knowledge Revisited- We Can Still Learn from Polanyi”, The Electronic Journal of Knowledge Management, Vol. 5 No. 2, pp. 173-180.

Harari, Y.N. (2016), Homo Deus: A brief history of tomorrow, Random House.

Hornecker, E. (2011), "The role of physicality in tangible and embodied interactions", Interactions, Vol. 18 No. 2, pp. 19-23.

Huxley, A. (1954 - 2004), The Doors of Perception and Heaven and Hell, Vintage, London.

Ishii, H. and Ullmer, B. (1997), "Tangible bits: towards seamless interfaces between people, bits and atoms", Proceedings of the ACM SIGCHI Conference on Human factors in computing systems, ACM, pp. 234-241.

Ishii, H., Ratti, C., Piper, B., Wang, Y., Biderman, A. and Ben-Joseph, E. (2004), "Bringing clay and sand into digital design-continuous tangible user interfaces", BT technology journal, Vol. 22 No. 4, pp. 287-299. 
Jacob, R.J., Girouard, A., Hirshfield, L.M., Horn, M.S., Shaer, O. et al. (2008), "Reality-based interaction: a framework for post-WIMP interfaces", Proceedings of the SIGCHI conference on Human factors in computing systems, ACM, pp. 201-210.

Jetter, H.C., Reiterer, H. and Geyer, F. (2014), "Blended Interaction: understanding natural human-computer interaction in post-WIMP interactive spaces", Personal and Ubiquitous Computing, Vol. 18 No. 5, pp. 1139-1158.

Johnson, M. (2013), The body in the mind: The bodily basis of meaning, imagination, and reason, University of Chicago Press.

Kierkegaard, S. (2012), The Soul of Kierkegaard: Selections from His Journals, Courier Corporation.

Kosmadoudi, Z., Lim, T., Ritchie, J.M., Liu, Y., Sung, R. et al. (2013), "Harmonizing Interoperability-Emergent Serious Gaming in Playful Stochastic CAD Environments", Games and Learning Alliance, Springer International Publishing, pp. 390-399.

Lakoff, G. and Johnson, M. (1980), Metaphors we live by, Chicago/London.

Lévi-Strauss, C. (1966), "Culinary triangle”, New Society, Vol. 8 No. 221, pp. 937-940.

Marquard, O. (1991), In defense of the accidental: Philosophical studies, Oxford University Press on Demand.

Polanyi, M. (1966), The tacit dimension, The University of Chicago Press, Chicago and London.

Robertson, B.F. and Radcliffe, D.F. (2009), "Impact of CAD tools on creative problem solving in engineering design", Computer-Aided Design, Vol. 41 No. 3, pp. 136-146.

Ros, F. (2015), Stewart. [online] Available at: http:/felixros.com/stewart.html (accessed 09.12.2017).

Schaefer, P.M. (2016), Tangible Pods: A raw tangible user interface (TUI) for audio and haptic feedback, Bachelor's thesis, University of Twente.

Schön, D.A. (1983), The reflective practitioner: How professionals think in action, Basic books.

Schön, D.A. (1992), "Designing as Reflective Conversation with the Materials of a Design Situation", Research in Engineering Design, Vol. 3 No. 3, pp. 131-147.

Sennett, R. (2008), The craftsman, Yale University Press.

Shaer, O. and Hornecker, E. (2010), "Tangible user interfaces: past, present, and future directions", Foundations and Trends in Human-Computer Interaction, Vol. 3 No. 1-2, pp. 1-137.

Spool, J.M. (2005), What Makes a Design Seem 'Intuitive'? User Interface Engineering. [online] Available at: http://www.uie.com/articles/design_intuitive/ (accessed 09.12.2017).

Terken, J., Levy, P., Wang, C., Karjanto, J., Yusof, N.M. et al. (2017), "Gesture-based and haptic interfaces for connected and autonomous driving", Advances in Human Factors and System Interactions. Advances in Intelligent Systems and Computing, Vol. 497, Springer, Cham.

Ullmer, B.A. (1997), Models and mechanisms for tangible user interfaces, $\mathrm{PhD}$ thesis, MIT.

Valli, A. (2006), “The Design of Natural Interaction", Multimedia Tools and Applications, Vol. 38 No. 3, pp. $205-305$.

Van Dam, A. (1997), "Post-WIMP user interfaces", Communications of the ACM, Vol. 40 No. 2, pp. 63-67.

Weiser, M. (1993), "Ubiquitous computing”, Computer, Vol. 26 No. 10, pp. 71-72.

Wendrich, R.E. (2010), "Raw Shaping Form Finding: Tacit Tangible CAD", Computer- Aided Design \& Applications, Vol. 7 No. 4, pp. 505-531.

Wendrich, R.E., Tragter, H., Kokkeler, F.G.M. and van Houten, F.J.A.M. (2009), "Bridging the Design Gap: Towards an Intuitive Design Tool", Proceedings of the 26th ICSID World Design Congress and Education Congress, Singapore.

Wendrich, R.E. (2016), Hybrid design tools for conceptual design and design engineering processes: bridging the design gap: towards an intuitive design tool, $\mathrm{PhD}$ thesis, Universiteit Twente.

Wendrich, R.E. (2012), "Multimodal Interaction, Collaboration, and Synthesis in Design and Engineering Processing", Proceedings of DESIGN 2012, the 12th International Design Conference, Dubrovnik, Croatia, pp. 579-588.

Wendrich, R.E. (2014), "Hybrid Design Tools For Design and Engineering Processing", In: Michopoulos, J., Rosen, D., Paredis, C. and Vance, J. (Eds.), Advances in Computers and Information in Engineering Research (ACIER), American Society of Mechanical Engineers (ASME), NewYork, NY, USA, pp. 215-23

Wendrich, R.E. (2016), "Blended Spaces for Integrated Creativity and Play in Design and Engineering Processes", ASME Journal of Computing and Information Science in Engineering (JCISE), Vol. 16 No. 3, pp. 030905. https://doi.org/10.1115/1.4033217

Whitehead, A.N. and Sherburne, D.W. (1957), Process and reality, Macmillan, New York, NY.

Dr. Robert Wendrich, Ing.

University of Twente, Design, Production \& Management

Drienerlolaan 5, 7522NB Enschede, Netherlands

Email: r.e.wendrich@utwente.nl 